\title{
Mudanças nos cursos de Odontologia e a interação com o SUS
}

\author{
O conceito de saúde explicitado na Constituição e os princípios que \\ nortearam a criação e implantação do SUS são fundamentais na \\ definição das Diretrizes Curriculares dos cursos da área de Saúde.
}

\author{
Maria Celeste Morita*, Léo Kriger** \\ * Professora Associada da Universidade Estadual de Londrina. \\ ** Presidente da Comissão de Ensino da ABENO.
}

A s Diretrizes Curriculares Nacionais (DCN) são orientações para elaboração dos currículos que devem ser necessariamente adotadas por todas as instituições de ensino superior. Na área da Saúde, entretanto, estas precisam ser entendidas dentro de um contexto maior, o da Reforma Sanitária Brasileira.

A Reforma Sanitária Brasileira, movimento social amplo que traz entre seus pontos estratégicos a criação do Sistema Único de Saúde (SUS), pretende garantir a saúde como um direito do cidadão.

Embora tenham ocorrido movimentos significativos para uma reflexão crítica sobre os modelos tradicionais de formação profissional em várias áreas da Saúde, principalmente na Medicina e na Enfermagem, em relação à Odontologia, existe um atraso histórico destes movimentos de mudança, exigindo daqui para a frente um esforço redobrado para que possamos integrar a saúde bucal dentro do novo contexto de ação interdisciplinar e multiprofissional, formando um profissional com perfil adequado ${ }^{10}$.

Advindo de um processo de construção coletiva, através das Conferências Nacionais de Saúde, o capítulo da Constituição Brasileira que define o SUS traz também um marco regulatório:

\footnotetext{
"Compete à gestão do Sistema Único de Saúde o ordenamento da formação de recursos humanos da área da Saúde, bem como o incremento, na sua área de atuação, do desenvolvimento científico e tecnológico" (Constituição Federal, Art. 200, incisos III e IV) ${ }^{3}$.
}

Apesar deste preceito constitucional, ele não tem se constituído em prática institucional. Somente recentemente, o Ministério da Saúde tem se preocupado em orientar o processo de formação dos recursos humanos da área, estabelecendo para tanto parceria com o Ministério da Educação. A nova definição política do Ministério da Saúde, visando a diminuição do distanciamento entre a formação de recursos humanos e as necessidades do SUS, apontam para a necessidade da ABENO definir uma estratégia de trabalho, que vise principalmente a aproximação da Odontologia às demais áreas da Saúde.

Todos os esforços prévios de integração do processo de ensino-aprendizagem à rede de serviços tiveram pouca sustentabilidade, pois sempre dependeram de uma adesão ideológica de docentes e estudantes. As atividades de ensino-aprendizagem, extramurais, sempre dependeram mais da voluntariedade dos professores que as coordenavam do que do apoio institucional e da participação do quadro docente como um todo.

No estágio atual, embora o SUS constitua um significativo mercado de trabalho para os profissionais da Odontologia, principalmente com a inserção da saúde bucal na Estratégia de Saúde da Família, este fato ainda não tem sido suficiente para produzir o impacto esperado sobre o ensino de graduação.

Para garantir uma efetiva integração, o conceito de saúde explicitado na Constituição e os princípios que nortearam a criação e implantação do SUS foram elementos fundamentais na definição das Diretrizes Curriculares dos cursos da área da Saúde. Do ponto de vista legal, o SUS precisa ser entendido como um 
interlocutor essencial das escolas na formulação e implementação dos projetos pedagógicos de formação profissional e não mero campo de estágio ou aprendizagem prática.

Esta articulação está claramente explicitada no objeto das Diretrizes:

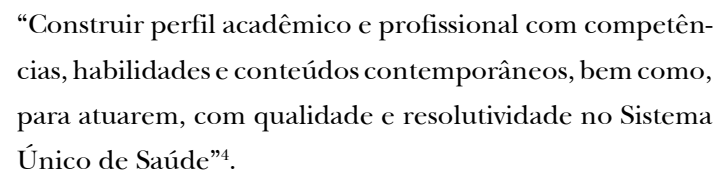

Nas DCN de Odontologia de fevereiro de 2002, também se destaca:

\footnotetext{
"A formação do cirurgião-dentista deverá contemplar o sistema de saúde vigente no país, a atenção integral da saúde no sistema regionalizado e hierarquizado de referência e contra-referência e o trabalho em equipe (Resolução CES/CNE nº 03 - DCN/Odontologia)" ${ }^{5}$.
}

Em uma rede de inter-relações indissociáveis entre saúde e educação, as Diretrizes Curriculares assumem um papel estratégico no aperfeiçoamento do SUS. Ao mesmo tempo, a discussão sai dos limites acadêmicos a medida que a sociedade começa a exigir melhores serviços de saúde.

Afinal, se pergunta: Quais as competências e habilidades necessárias para o trabalho no SUS?

Para trabalhar no SUS com qualidade e atender as necessidades da população, é necessário ser um profissional generalista tecnicamente competente e com sensibilidade social. As Diretrizes, portanto, valorizam além da excelência técnica a relevância social das ações de saúde e do próprio ensino. Sem dúvida isso implica na formação de profissionais capazes de prestar atenção integral mais humanizada, trabalhar em equipe e compreender melhor a realidade em que vive a população.

A inserção da saúde bucal na Estratégia de Saúde da Família, por exemplo, tem como uma de suas prioridades a atenção básica à saúde. Tal proposta, no entanto, defronta-se com a precária disponibilidade de profissionais de Saúde dotados de visão humanística e preparados para prestar cuidados contínuos e resolutivos à comunidade, funcionando como porta de entrada do sistema de saúde.

Em síntese, todas as habilidades e competências gerais e específicas descritas nas DCN são necessárias para o SUS. Uma leitura menos atenta pode dar a impressão de que as Diretrizes são discursivas e que apontam para atividades que já estão presentes nos projetos pedagógicos dos cursos de Odontologia. Esta porém, é uma visão equivocada sendo muito importante compreender que a implementação das Diretrizes Curriculares deve significar um avanço dos projetos pedagógicos e não uma acomodação de situações existentes. Destacamos para reflexão, algumas competências e habilidades que certamente necessitarão de uma nova abordagem das escolas de Odontologia. No artigo $4^{\circ}$, o cirurgião-dentista deve ser capaz de ${ }^{5}$ :

- pensar criticamente;

- tomar decisões;

- ser líder;

- atuar em equipes multiprofissionais;

- planejar estrategicamente para contínuas mudanças;

- administrar e gerenciar serviços de saúde;

- aprender permanentemente.

Surge então, a seguinte questão: quais são as experiências educativas que os alunos de Odontologia vivenciam para tanto? Nossos projetos pedagógicos estão adequados para esta formação? Apenas para exemplo, a capacidade de liderança necessita de algumas habilidades que só podem ser desenvolvidas em oportunidades práticas. Não basta dizer que se espera que o profissional de Odontologia seja capaz de assumir a liderança na equipe de saúde. Ele tem que ter tido durante a sua formação oportunidades concretas para desenvolver esse papel.

No artigo $5^{\circ}$, o cirurgião-dentista deve ser capaz de: "atuar multiprofissionalmente, interdisplinarmente e transdisciplinarmente com extrema produtividade na promoção da saúde baseado na convicção científica, de cidadania e de ética"5.

O texto das DCN reforça nitidamente a importância do trabalho em equipes multiprofissionais, entendendo que "a equipe de saúde como um todo tem maior impacto do que a soma das contribuições dos membros de forma isolada" 12 .

Atuar multiprofissionalmente significa romper o modelo centrado no trabalho individual capacitando para o trabalho em equipe onde se propõe compartilhar o planejamento, a divisão de tarefas, aprender a cooperar e colaborar. Parte-se da compreensão que os problemas de saúde são sempre interdisciplinares. Para entender melhor o alcance dessa interação, dois conceitos precisam ser aprofundados: a interdisciplinaridade e a transdisciplinaridade.

Segundo Piaget, a interdisciplinaridade, é

\footnotetext{
"o nível em que a interação entre várias disciplinas ou se-
} tores heterogêneos de uma mesma ciência conduz a inte- 
rações reais, a uma certa reciprocidade no intercâmbio levando a um enriquecimento mútuo"9.

$\mathrm{Na}$ transdisciplinaridade, o conceito envolve

“não só as interações ou reciprocidade entre projetos especializados de pesquisa, mas a colocação dessas relações dentro de um sistema total, sem quaisquer limites rígidos entre as disciplinas"9.

Portanto, é na compreensão do conceito de saúde, na possibilidade de se formar um profissional capaz de fazer uma contribuição permanente para a produção da saúde da sociedade em que vive que as Diretrizes se amplificam.

Como se vê, uma leitura crítica das DCN nos leva à reflexão de que profundas mudanças são necessárias nos cursos de graduação em Odontologia para formar profissionais adequados às necessidades de saúde da população e do SUS.

Para que esse processo avance é essencial que ocorra efetiva articulação entre as políticas de Educação e Saúde. A inadequação da formação dos profissionais de Saúde tem levado à necessidade de requalificação profissional que podem ser constatadas através dos recursos gastos pelo Ministério da Saúde em "capacitações”. A implantação das Diretrizes Curriculares assume, portanto, grande importância no aperfeiçoamento do SUS.

Na agenda estratégica dos Ministérios da Saúde e de Educação destacam-se dois pontos fundamentais: o incentivo à implementação das Diretrizes Curriculares e o uso da avaliação como estratégia para fortalecer as mudanças.

Estas prioridades estão ligadas à ineficiência do modelo formador vigente, como indica o documento que trata da Política de Educação e Desenvolvimento para o SUS:

\footnotetext{
"A formação tradicional em saúde, baseada na organização disciplinar e nas especialidades, conduz ao estudo fragmentado dos problemas de saúde das pessoas e das sociedades, levando à formação de especialistas que não conseguem mais lidar com as totalidades ou com realidades complexas"7.
}

Para formar profissionais com o perfil que atenda as necessidades do SUS os cursos de saúde precisam:

1. Adequar sua abordagem pedagógica.

2. Favorecer a articulação dos conhecimentos.

3. Trabalhar em equipes multiprofissionais.
4. Promover atividades práticas ao longo de todo o curso em todos os tipos de unidades de saúde.

Para auxiliar neste processo, os cursos poderão estabelecer protocolos de cooperação sistemática com a ABENO, o Ministério da Saúde, o Ministério da Educação e os gestores estaduais e municipais do SUS:

1. Na abordagem pedagógica tradicional observa-se uma baixa eficácia, os conteúdos se apresentam distantes da realidade e das necessidades de aprendizagem o que leva ao desperdício de tempo, de esforços e à necessidade de requalificação ${ }^{7}$.

A mudança didático-pedagógica que se almeja visa sair do ensino centrado no professor para atingir uma aprendizagem ativa desenvolvendo-se em múltiplos cenários (DCN, Art. $7^{\circ}$ e 13, inciso III). O novo modelo pedagógico deve contemplar o equilíbrio entre a excelência técnica e a relevância social. Cabe ao professor o papel de facilitador do processo de construção do conhecimento, caracterizando-se o aluno como o sujeito da aprendizagem, com eficiente integração curricular.

2. Na articulação de conhecimentos, busca-se mudar a estrutura curricular de tal forma que se elimine os ciclos clínico e básico completamente separados e organizados em disciplinas fragmentadas, passando para um currículo majoritariamente integrado, sem disciplinas isoladas (DCN, Art. 13, incisos II e III). Este processo pretende a articulação e o diálogo entre atores e saberes da clínica, da saúde coletiva e da gestão a partir das necessidades locais.

3. $\mathrm{O}$ trabalho em equipes multiprofissionais deve se dar entre profissionais de uma mesma disciplina ou carreira (equipe de saúde bucal: THD, ACD, e CD) bem como com os demais profissionais da Saúde.

4. Um outro ponto fundamental, diz respeito aos cenários de aprendizagem.

$\mathrm{Na}$ maior parte das instituições, as clínicas de ensino, principalmente as voltadas para as especialidades, continuam sendo o local privilegiado para o treinamento prático dos estudantes. No entanto, os movimentos atuais de reorganização da prática clínica odontológica indicam uma tendência progressiva à antecipação das clínicas integradas, com complexidade crescente, envolvendo o aluno desde os primeiros anos na visão integral do paciente. Práticas de ensino também podem ser desenvolvidas, com eficácia e eficiência, em Unidades Básicas de Saúde, na comunidade e nos domicílios, permitindo ao aluno um melhor conhecimento da população que será atendida. Os cenários de ensino, portanto, devem ser diversifi- 
cados, agregando-se ao processo, além dos equipamentos de saúde, os equipamentos educacionais e comunitários.

A interação ativa do aluno com a população e profissionais de Saúde deve ocorrer desde o início do processo de formação, trabalhando com problemas reais, assumindo responsabilidades crescentes.

Para entender a rede de serviços como espaço privilegiado de ensino é preciso partir de um conceito ampliado de saúde onde todos os cenários em que se produz saúde são ambientes relevantes de aprendizagem $^{1}$. Portanto, na diversificação dos cenários de ensino-aprendizagem é preciso sair das práticas profissionalizantes realizadas em clínicas de ensino de especialidades para as práticas profissionalizantes em clínicas integradas e atividades extramurais em unidades do SUS, com graus crescentes de complexidade. Bem como, substituir os serviços próprios isolados da rede SUS, por serviços próprios completamente integrados ao SUS, com desenvolvimento de mecanismos institucionais de referência e de contra-referência com a rede SUS. A evolução do SUS gerou uma gama de oportunidades de aprendizagem que levaram o Ministério da Saúde, a desenvolver um projeto de Vivências e Estágios na Realidade do Sistema Único de Saúde do Brasil, o VER-SUS/Brasil ${ }^{6}$. No projeto, alunos de diferentes carreiras estagiam em setores como o de financiamento, estratégias de controle e auditoria, formulação de políticas técnicas, ordenamento do trabalho e da rede assistencial, interpretação demográfica e epidemiológica, descentralização da gestão (compreensão das diferentes esferas de gestão) e exercício do controle social sobre o setor saúde em municípios de gestão plena da saúde.

As Diretrizes Curriculares fazem parte de um processo de superação de limites da formação e das práticas clínicas tradicionais. O longo percurso percorrido pela ABENO nos mostra que os pilares dessas mudanças têm sido discutidos nas reuniões anuais. Se analisarmos os textos já produzidos ${ }^{8}$, os temas de reuniões ${ }^{2}$, veremos que há grande congruência de objetivos. Queremos a melhoria da qualidade do ensino de Odontologia. A construção progressiva das Diretrizes pode também ser observada em outros documentos anteriores ${ }^{11}$. Não nos são estranhas nenhumas destas afirmações: a importância da inserção precoce do aluno em seu contexto profissional, o currículo integrado, as clínicas integradas de complexidade crescente, a melhoria da formação em Saúde Coletiva, ganhos do trabalho em equipe e diversificação dos cenários de aprendizagem ${ }^{3}$. Dois objetivos não pode- rão ser perdidos de vista na implementação das DCN: a integração curricular e a integralidade da atenção.

Em conclusão, as Diretrizes indicam a necessidade de transformar o modelo de atenção, fortalecendo a promoção e prevenção, oferecendo atenção integral e fortalecendo a autonomia dos sujeitos na produção da saúde.

O grande desafio está em sair de um modelo de ensino centrado no diagnóstico, tratamento e recuperação de doenças para outro centrado na promoção de saúde, prevenção e cura de pessoas.

\section{REFERÊNCIAS BIBLIOGRÁFICAS}

1. ABENO. Comissão de Ensino. Diretrizes da ABENO para a definição do Estágio Supervisionado nos cursos de Odontologia. [citado 2004 abr 21] Disponível em: http://www.abeno.org. $b r$.

2. ABENO - XXXIII Reunião da Associação Brasileira de Ensino Odontológico. Diretrizes Curriculares: o que muda? Rev ABENO 1999;3-6.

3. Brasil. Constituição da República Federativa do Brasil. Brasília: Senado Federal; 1988.

4. Brasil. Ministério da Educação. Parecer n ${ }^{\circ}$ CNE/CES 1300/01 de 06 de novembro de 2001. Institui as Diretrizes Curriculares Nacionais dos Cursos de Farmácia e Odontologia. Diário Oficial, Brasília, 07 dez 2001, seção 1, p. 25.

5. Brasil. Ministério da Educação. Resolução n ${ }^{\circ}$ CNE/CES 3/2002 de 19 de fevereiro de 2002. Institui as Diretrizes Curriculares Nacionais dos Cursos de Farmácia e Odontologia. Diário Oficial, Brasília, 04 mar 2002, seção 1, p. 10.

6. Brasil. Ministério da Saúde. Políticas de Formação e Desenvolvimento para o SUS: caminhos para a educação permanente em saúde. [citado 2004 abr 20] Disponível em: http://portal. saude.gov.br/saude/arquivos/pdf/pol_formacao_desenv.pdf.

7. Brasil. Ministério da Saúde/Secretaria de Gestão do Trabalho e da Educação na Saúde. Caminhos para as mudanças da formação e desenvolvimento dos profissionais de Saúde: diretrizes para a ação política para assegurar educação permanente no SUS. Brasília, maio 2003. (mimeografado)

8. Carvalho ACP, Fernandes Neto AJ. Recomendações sobre a implementação das Diretrizes Curriculares Nacionais dos cursos de graduação em Odontologia. ABENO: Uberlândia; 2001. (mimeografado)

9. Chaves M. Complexidade e transdiciplinariedade: uma abordagem multidimensional do Setor Saúde. [citado 2004 jun 13] Disponível em: http://psy.med.br/textos/complexidade/complexidade.pdf.

10. Paraná. Escola de Saúde Pública. Programa de Incentivo a Mudanças nos Cursos de Medicina, Odontologia, Enfermagem e Farmácia do Estado do Paraná. [citado 2004 abr 30] Disponível em: http://www.redeunida.org.br/prosaudepr.asp. 
11. Perri de Carvalho AC. Formação do cirurgião-dentista - ensino e profissionalização. Documento de trabalho 4/96. São Paulo: NUPES/USP; 1996.

12. World Health Organization. Learning together to work together for health. Report of WHO Study Group on Multiprofes- sional Education for Health Personnel: the team approach. Technical Report Series 769. WHO: Geneva; 1988.

Aceito para publicação em 11/2003

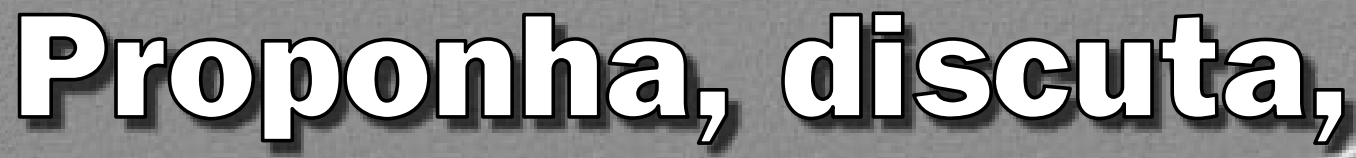
paricipel
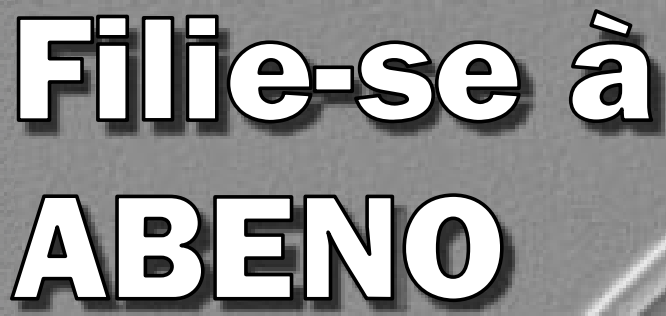

\section{Ensino Odontológico tem como OBJETIVOS:}

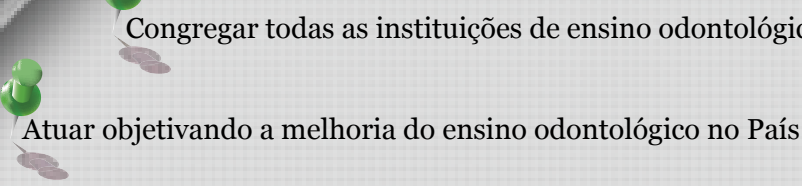

Adotar medidas que objetivem a formação e o aperfeiçoamento

do pessoal docente e dos profissionais de Odontologia

Estimular as atividades de pesquisa na ciência odontológica

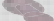

Incentivar as atividades de extensão e de educação em saúde junto às comunidades

Defender os interesses das instituições de ensino que a integram

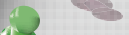

Constituir-se fator de integração entre o ensino e a cultura nacional

\section{Associação Brasileira de} $-1$

Manter relações com as entidades representativas da categoria odontológica 13

Manter intercâmbio com entidades estrangeiras representativas da docência odontológica

Filiando-se à ABENO, vocề estará inserido no processo de melhoria do ensino e do exercício da Odontologia no Brasil. 